\title{
Milnor-Wood inequalities for manifolds locally isometric to a product of hyperbolic planes
}

\author{
Michelle Bucher ${ }^{\mathrm{a}}$, Tsachik Gelander ${ }^{\mathrm{b}}$ \\ ${ }^{a}$ KTH Mathematics Department, 10044 Stockholm, Sweden \\ ${ }^{\mathrm{b}}$ Einstein Institute of Mathematics, The Hebrew University, Jerusalem, 91904, Israel
}

\begin{abstract}
This note describes sharp Milnor-Wood inequalities for the Euler number of flat oriented vector bundles over closed Riemannian manifolds locally isometric to products of hyperbolic planes. One consequence is that such manifolds do not admit an affine structure, confirming Chern-Sullivan's conjecture in this case. The manifolds under consideration are of particular interest, since in contrary to many other locally symmetric spaces they do admit flat vector bundle of the corresponding dimension. When the manifold is irreducible and of higher rank, it is shown that flat oriented vector bundles are determined completely by the sign of the Euler number.
\end{abstract}

\section{Résumé}

Inégalités de Milnor-Wood pour variétés localement isométriques à un produit de plans hyperboliques. Nous généralisons l'inégalité classique de Milnor aux variétés localement isométriques à un produit de plans hyperboliques. Il en découle que de telles variétés n'admettent pas de structure affine, confirmant dans ce cas la conjecture de Chern-Sullivan. Contrairement à de nombreuses variétés localement symétriques, les variétés considérées dans cette note admettent un fibré vectoriel plat en dimension correspondante. Si les variétés sont de plus irréductibles de rang supérieur, nous montrons qu'un fibré vectoriel orienté plat avec nombre d'Euler non nul est, à orientation près, unique.

Version française abrégée

Soit $\xi$ un $\mathrm{GL}^{+}(m, \mathbb{R})$-fibré principal, ou de façon équivalente, un fibré vectoriel orienté de fibre $\mathbb{R}^{m}$, sur une variété fermée $M$ orientée de dimension $m$. Rappelons que la classe d'Euler (réelle) de $\xi$ est la classe de cohomologie $\varepsilon_{m}(\xi) \in H^{m}(M, \mathbb{R})$ qui est l'image, par l'inclusion de coefficients $\mathbb{Z} \hookrightarrow \mathbb{R}$, de l'obstruction

Email addresses: mickar@math.kth.se (Michelle Bucher), tsachik.gelander@gmail.com (Tsachik Gelander). 
à l'existence d'une section non nulle dans le fibré vectoriel (associé). Le nombre d'Euler de $\xi$ est le produit de Kronecker de la classe d'Euler avec la classe fondamentale (réelle) $[M] \in H_{m}(M, \mathbb{R})$ de $M$ :

$$
\chi(\xi)=\left\langle\varepsilon_{m}(\xi),[M]\right\rangle .
$$

Si $\xi$ admet une structure plate, c'est-à-dire si $\xi$ est induit par une représentation $\rho: \pi_{1}(M) \rightarrow \mathrm{GL}^{+}(m, \mathbb{R})$ du groupe fondamental de $M$, alors il existe une borne sur $|\chi(\xi)|$ ne dépendant que de $M$. En effet, c'est une observation de Lusztig, que l'espace des représentations de $\pi_{1}(M)$ dans $\mathrm{GL}^{+}(m, \mathbb{R})$ est une variété algébrique, et n'a en conséquence qu'un nombre fini de composantes connexes. Comme le nombre d'Euler est constant sur les composantes connexes, l'affirmation s'ensuit.

Dénotons par $X$ l'espace hyperbolique réel. Milnor, en premier, a exhibé des fibrés plats dont la classe d'Euler est non nulle, et de plus, donné une borne optimale pour le nombre d'Euler de fibrés plats au dessus de surfaces hyperboliques [8]. Nous généralisons cette inégalité aux variétés localement isométriques à un produit de plans hyperboliques $X^{n}$, le cas $n=1$ étant l'inégalité de Milnor.

Theorem 2.1 Soit $M$ une variété Riemannienne fermée localement isométrique à $X^{n}$ et soit $\xi$ un $\mathrm{GL}^{+}(2 n, \mathbb{R})$-fibré sur $M$. Si $\xi$ admet une structure plate, alors

$$
|\chi(\xi)|=\left|\left\langle\varepsilon_{2 n}(\xi),[M]\right\rangle\right| \leq \frac{1}{2^{n}}|\chi(M)| .
$$

Il est immédiat que si l'espace tangent $T M$ d'une variété Riemannienne fermée $M$ localement isométrique à $X^{n}$ admettait une structure plate, l'inégalité $|\chi(M)|=|\chi(T M)| \leq\left(1 / 2^{n}\right)|\chi(M)|$ découlerait du Théorème 2.1, ce qui n'est pas possible puisque $\chi(M) \neq 0$. Comme une structure affine est clairement plate, nous en déduisons une réponse affirmative partielle à la conjecture de Chern-Sullivan prédisant qu'une variété fermée avec charactéristique d'Euler non nulle n'admet pas de structure affine :

Corollary 2.2 Une variété Riemannienne fermée localement isométrique à $X^{n}$ n'admet pas de structure affine.

Ce résulat est nouveau pour $n>2$. Pour $n=1$ et $n=2$ cela s'obtient déjà par les inégalités correspondantes dans [8] et [1] respectivement. L'inexistence de structure affine complète, qui suivrait aussi de la conjecture d'Auslander, est démontrée dans [7]. Notons que même dans le cas d'un produit de surfaces, l'inexistence de structure affine ne découle pas directement de l'inexistence de structure affine sur les surfaces. En effet, Etienne Ghys nous a montré un exemple d'une variété produit admettant une structure affine sans qu'aucun des facteurs n'en admettent une.

A revêtement fini près, les inégalités du Théorème 2.1 sont optimales, c'est-à-dire que pour toute variété fermée $M$ localement isométrique à $X^{n}$, il existe un $\mathrm{GL}^{+}(2 n, \mathbb{R})$-fibré $\xi$ admettant une structure plate sur un revêtement $N$ fini de $M$ et $\chi(\xi)=\left(1 / 2^{n}\right)|\chi(N)|$. Par contre, on ne peut pas réaliser chaque entier dans l'intervalle $\left[\frac{-|\chi(M)|}{2^{n}}, \frac{|\chi(M)|}{2^{n}}\right]$ comme nombre d'Euler de fibré plat. En effet, nous pouvons raffiner le théorème 2.1 comme dans le Théorème 2.3 ci-dessous. Le cas extrême étant quand $M$ est une variété que nous appellerons rigide, c'est-à-dire que $M$ n'admet pas de revêtement fini se décomposant en un produit contenant un facteur de dimension 2. Dans ce cas, les seules valeurs possibles pour le nombre d'Euler d'un $\mathrm{GL}^{+}(2 n, \mathbb{R})$-fibré $\xi$ sur $M$ admettant une structure plate sont 0 et $\pm\left(1 / 2^{n}\right)|\chi(M)|$. De plus, si le nombre d'Euler du fibré plat est non nul, alors la structure plate de $\xi$ est à orientation près unique. Voir Théorème 2.4 pour plus de détails.

Observons enfin que contrairement au cas de dimension 2, il n'est pas possible de caractériser les fibrés plats sur les variétés localement isométriques à $X^{n}$ en fonction de leur nombre d'Euler, car la classe d'Euler n'est pas un invariant complet de classes d'isomorphies de $\mathrm{GL}^{+}(2 n, \mathbb{R})$-fibrés en dimension supérieure. En effet, il n'est pas difficile de construire deux fibrés avec même nombre d'Euler au dessus de variétés localement $X^{n}$, pour $n>1$, telle que l'un des fibré admette une structure plate, et l'autre non. 


\section{Historical introduction}

The first example of a nontrivial characteristic class of flat bundle was given by Milnor in [8], where he characterized the $\mathrm{GL}^{+}(2, \mathbb{R})$-bundles admitting flat structures over surfaces in terms of their Euler number: $\mathrm{A} \mathrm{GL}^{+}(2, \mathbb{R})$-bundle $\xi$ over a surface $\Sigma_{g}$ of genus $g \geq 1$ admits a flat structure if and only if its Euler number $\chi(\xi)$ satisfies the inequality $|\chi(\xi)|=\left|\left\langle\varepsilon_{2}(\xi),\left[\Sigma_{g}\right]\right\rangle\right| \leq g-1$. Milnor's inequality was later generalized to circle bundles by Wood [11].

In his groundbreaking essay [4], Gromov naturally puts Milnor's inequality in the context of bounded cohomology. Indeed, canonical $L^{1}$ and $L^{\infty}$ norms can be defined on the spaces of singular chains and cochains of a closed oriented $n$-dimensional manifold $M$. These in turn induce seminorms on the respective real valued homologies and cohomologies. It follows from Hahn-Banach theorem that

$$
|\langle\beta,[M]\rangle|=\|\beta\|_{\infty} \cdot\|M\|, \forall \beta \in H^{n}(M) \text { with }\|\beta\|_{\infty}<\infty,
$$

where $\|M\|$ denotes the $L^{1}$ seminorm of the fundamental class of $M$, the so called simplicial volume of $M$. Thus, if $\beta$ is a characteristic class, a bound on the characteristic number $|\langle\beta,[M]\rangle|$ can be obtained by bounding both $\|\beta\|_{\infty}$ and $\|M\|$. Unfortunately, estimating each of these terms is usually very difficult.

Nonzero exact simplicial volume computations are rare. For oriented surfaces $\Sigma_{g}$ of genus $g \geq 1$, it is not difficult to show that $\left\|\Sigma_{g}\right\|=2\left|\chi\left(\Sigma_{g}\right)\right|=4(g-1)$. In particular, if $g \geq 2$ and $\Sigma_{g}$ is endowed with a hyperbolic structure, then $\left\|\Sigma_{g}\right\|=\pi \cdot \operatorname{Vol}\left(\Sigma_{g}\right)$. More generally, if $M$ is an $n$-dimensional closed hyperbolic manifold, then $\|M\|=v_{n} \cdot \operatorname{Vol}(M)[4,10]$, where $v_{n}$ denotes the supremum of the volumes of geodesic simplices in the $n$-dimensional hyperbolic space and is known explicitly in low dimensions only. The only further computation of a nonzero simplicial volume is given in [1] for manifolds locally isomorphic to the product of two copies of the hyperbolic plane. In this case, one has $\|M\|=6 \cdot \chi(M)=3 /\left(2 \pi^{2}\right) \cdot \operatorname{Vol}(M)$.

It is known since [4] that characteristic classes of flat $G$-bundles have finite $L^{\infty}$ seminorm when $G$ is a real algebraic subgroup of $\mathrm{GL}(n, \mathbb{R})$, but actual upper bounds for their norms are only known in special cases. For the Euler class $\varepsilon_{n}$, Gromov [4] obtained from Sullivan-Smillie's corresponding simplicial results that $\left\|\varepsilon_{n}(\xi)\right\|_{\infty} \leq 1 / 2^{n}$, whenever $\xi$ is a $\mathrm{GL}^{+}(n, \mathbb{R})$-bundle admitting a flat structure. Independently, Ivanov and Turaev [6] exhibited an explicit bounded cocycle representing the Euler class of flat bundles, producing the same bound. In degree 2, sharp upper bounds for the Kähler class were computed by Domic and Toledo [3] in terms of the rank of the associated symmetric space, later generalized by Clerc and Ørsted [2] to include all Hermitian symmetric spaces.

In view of the (im)possible seminorms computations, sharp generalizations of Milnor's inequality were essentially carried through in degree 2 only. In this note, we announce some new sharp upper bounds for the Euler number in degree $2 n$ of every flat $\mathrm{GL}^{+}(2 n, \mathbb{R})$-bundle $\xi$ over a closed manifold $M$ which admits a Riemannian structure locally isometric to a product of $n$ hyperbolic planes. Note however that our approach avoids estimating the simplicial volume $\|M\|$ as well as the norm of the Euler class. Instead, since by (1), $\|M\|=|\chi(M)| /\left\|\varepsilon_{2 n}(T M)\right\|_{\infty}$, we prove Theorem 2.1 by showing that $\left\|\varepsilon_{2 n}(\xi)\right\|_{\infty} \leq$ $\left(1 / 2^{n}\right)\left\|\varepsilon_{2 n}(T M)\right\|_{\infty}$ when $\xi$ is flat.

\section{Statement of results}

Denote by $X$ the real hyperbolic plane. We prove Milnor-Wood type inequalities for manifolds admitting a Riemannian structure for which the universal cover is isometric to the product of $n$ hyperbolic planes, in short, $X^{n}$-manifolds. The case $n=1$ is Milnor's classical inequality.

Theorem 2.1 Let $M$ be a closed $X^{n}$-manifold and $\xi$ a $\mathrm{GL}^{+}(2 n, \mathbb{R})$-bundle over $M$. If $\xi$ admits a flat structure, then 


$$
|\chi(\xi)|=\left|\left\langle\varepsilon_{2 n}(\xi),[M]\right\rangle\right| \leq \frac{1}{2^{n}}|\chi(M)| .
$$

It is an old conjecture of Chern (known also as the Chern-Sullivan conjecture), formulated independently by Milnor [8], that a closed manifold of even dimension with nonzero Euler characteristic cannot admit an affine structure. As a consequence of Theorem 2.1 we derive the following partial affirmative answer:

Corollary 2.2 A closed $X^{n}$-manifold does not admit an affine structure.

In fact, we obtain the stronger statement that the tangent bundle $T M$ of a closed $X^{n}$-manifold does not admit a flat structure. Indeed, if it had, Theorem 2.1 would yield the impossible inequality

$$
|\chi(M)|=|\chi(T M)|=|\langle\varepsilon(T M),[M]\rangle| \leq\left(1 / 2^{n}\right)|\chi(M)| .
$$

Note that in general manifolds with nonzero Euler characteristic may admit a flat structure (cf. Smillie $[9])$.

The $X^{n}$-manifolds are of particular interest among all locally symmetric manifolds of even dimension. Indeed, while one can deduce directly from Margulis superrigidity theorem that many higher rank irreducible locally symmetric manifolds $M$ admit no flat bundle of dimension $\operatorname{dim}(M), X^{n}$-manifolds do admit (in many cases a unique) such flat bundle (cf. Theorems 2.3 and 2.4). By Theorem 2.1 these bundles cannot be isomorphic to the tangent bundle $T M$.

Corollary 2.2 is new for $n>2$. For $n=1$ and $n=2$ it follows from the corresponding inequalities in [8] and [1] respectively. The nonexistence of a complete affine structure is proved in [7]. However, proving the nonexistence of a non-complete affine structure is usually much harder. Note also that even in the case of products of hyperbolic surfaces, the nonexistence of affine structures on the product can not be directly deduced from the nonexistence of affine structure on the factors. Etienne Ghys showed us an example of a product manifold which admits an affine structure, while none of the factors does.

It is easy to construct examples of flat bundles over $X^{n}$-manifolds. More precisely, for any closed locally $X^{n}$-manifold $M$, there exists a $\mathrm{GL}^{+}(2 n, \mathbb{R})$-bundle $\xi$ over a finite cover $N$ of $M$ such that $\xi$ admits a flat structure and $\chi(\xi)=\left(1 / 2^{n}\right)|\chi(N)|$. This shows that, up to finite cover, the inequality of Theorem 2.1 is sharp. However, not every integer in the interval $\left[\frac{-|\chi(M)|}{2^{n}}, \frac{|\chi(M)|}{2^{n}}\right]$ is in general an Euler number of a flat bundle, and Theorem 2.1 can be refined as in Theorem 2.3 below. We will say that an $X^{n}$-manifold is rigid if it has no finite cover which decomposes as a product manifold with a 2-dimensional factor. This terminology is motivated by the (local, Mostow and Margulis) rigidity theorems which apply for such manifolds.

Theorem 2.3 Let $M$ be a closed $X^{n}$-manifold, and $\xi$ a $\mathrm{GL}^{+}(2 n, \mathbb{R})$-bundle over $M$. Let $N$ be a finite covering of $M$ of the form

$$
N=\Sigma_{g_{1}} \times \ldots \times \Sigma_{g_{k}} \times N^{\prime},
$$

where $N^{\prime}$ is rigid, $k \geq 0$, and the $\Sigma_{g_{i}}$ 's are surfaces of genus $g_{i} \geq 2$. Let $\xi_{N}$ be the pullback of $\xi$ to $N$. If $\xi$ admits a flat structure, then so does $\xi_{N}$ and

$$
\chi\left(\xi_{N}\right) \in\left\{\frac{\left|\chi\left(N^{\prime}\right)\right|}{2^{\operatorname{Dim}\left(N^{\prime}\right)}} \Pi_{i=1}^{k} \ell_{i}:\left|\ell_{i}\right| \leq g_{i}-1\right\} \cup\{0\} .
$$

Moreover, upon passing to a finite cover of $N$, all the integers are actually attained as Euler numbers of flat bundles.

Note that contrarily to the 2-dimensional case, one cannot characterize flat bundles over closed $X^{n}$ manifolds by their possible Euler numbers, since the Euler class is not a complete invariant of isomorphism classes of $\mathrm{GL}^{+}(2 n, \mathbb{R})$-bundles in higher dimensions. Thus, the converses of Theorems 2.3 and 2.1 are in general not true. In contrast, for rigid manifolds the Euler number does characterize flat bundles, whenever it does not vanish: 
Theorem 2.4 Let $M$ be a rigid closed $X^{n}$-manifold and let $\xi$ be a $\mathrm{GL}^{+}(2 n, \mathbb{R})$-bundle over $M$. Assume that $\xi$ admits a flat structure. Then either $\chi(\xi)=0$ or $|\chi(\xi)|=(1 / 2)^{n}|\chi(M)|$. Moreover, if $\chi(\xi) \neq 0$, then $\chi(\xi)$ completely determines the bundle and the flat structure.

\section{About the proof of Theorem 2.1}

Our proof of Theorem 2.1 combines bounded cohomology, representation theory and rigidity theory.

Let $\xi$ be a $\mathrm{GL}^{+}(2 n, \mathbb{R})$-bundle with nonzero Euler number admitting a flat structure over a closed $X^{n}$-manifold $M$. Upon passing to a double cover of $M$ we can suppose that $M$ is oriented. The flat bundle $\xi$ is induced by a representation

$$
\rho: \pi_{1}(M) \longrightarrow \mathrm{GL}^{+}(2 n, \mathbb{R}) .
$$

Denote by $\varepsilon_{2 n} \in H^{2 n}\left(\prod_{i=1}^{n} \mathrm{GL}(2, \mathbb{R})^{+}\right)$the (universal) Euler class of flat bundles, and note that by naturality, the Euler class of $\xi$ is the pullback $\varepsilon_{2 n}(\xi)=\rho^{*}\left(\varepsilon_{2 n}\right)$ of $\varepsilon_{2 n}$ by $\rho$. The main part of our proof consists of several steps which allow us to reduce the general case, without changing the $L^{\infty}$ seminorm of the Euler class $\rho^{*}\left(\varepsilon_{2 n}\right)$, to the situation where $\operatorname{Im}(\rho)$ is contained in a direct product of $n$ diagonally embedded $G L^{+}(2, \mathbb{R})$ 's. We prove and make use of the following general lemma:

Lemma 3.1 Let $G_{i}$ be groups and let $\rho: \prod_{i=1}^{n} G_{i} \rightarrow \mathrm{GL}_{m}^{+}(\mathbb{R})$ be an orientable representation such that $\rho\left(G_{i}\right)$ is not amenable for each $i$. Then $m \geq 2 n$, and in case $m=2 n$, up to replacing each $G_{i}$ by $a$ finite index subgroup, $V=\mathbb{R}^{2 n}$ decomposes as a direct sum of $n$ invariant planes $V_{i}$ such that $\rho\left(G_{i}\right)$ acts irreducibly on $V_{i}$ and scalarely (i.e. by multiplications by scalar diagonal matrices) on each $V_{j}, j \neq i$.

Upon replacing $M$ by a finite cover, we can without loss of generality suppose that we are in one of the following three cases:

(i) $M$ is completely reducible, i.e. a finite cover of $M$ is a product of $n$ surfaces $\Sigma_{g_{1}} \times \ldots \times \Sigma_{g_{n}}$. In this case, we applying Lemma 3.1 directly to the product $\prod_{i=1}^{n} \pi_{1}\left(\Sigma_{g_{i}}\right)$ and conclude that if $\left\|\rho^{*}\left(\varepsilon_{2 n}\right)\right\| \neq$ 0 , then none of the $\rho\left(\pi_{1}\left(\Sigma_{g_{i}}\right)\right)$ 's is amenable and the representation factors, up to conjugation and upon replacing $\pi_{1}(M)$ by a finite index subgroup, through the diagonally embedded product $\Pi_{i=1}^{n} \mathrm{GL}^{+}(2, \mathbb{R})$.

(ii) $M$ is rigid, i.e. no finite cover of $M$ admits a two dimensional factor. Here, we use Margulis Superrigidity Theorem to show that $\rho\left(\pi_{1}(M)\right)$ is, up to finite index, contained in a connected semisimple Lie group $S$ for which all the noncompact simple factors are locally isomorphic to $\operatorname{PSL}(2, \mathbb{R})$. Furthermore, if $S$ has $n$ factors which are all noncompact, then we get from Lemma 3.1 applied to $S$ that $S$ is conjugated to the diagonally embedded product $\prod_{i=1}^{n} \mathrm{SL}(2, \mathbb{R})$.

(iii) $M$ is mixed, i.e. admits a finite cover which is a direct product of surfaces and a rigid manifold. In this last case, the image $\rho\left(\pi_{1}(M)\right)$ is contained in a semidirect product $S \ltimes A$, where $S$ is semisimple and $A$ is a connected amenable normal subgroup. Since $A$ is amenable, denoting by $p$ the projection $p: S \ltimes A \rightarrow S$, we get $\left\|\rho^{*}\left(\varepsilon_{2 n}\right)\right\|_{\infty}=\left\|\rho^{*}\left(p^{*}\left(\varepsilon_{2 n}\right)\right)\right\|_{\infty}$, and we can hence replace the representation $\rho$ by the composition of $\rho$ with the projection $p$. If for one of the factors $M_{i}$ of $M$, the image $\rho\left(\pi_{1}\left(M_{i}\right)\right)$ lies in a closed amenable group, or in the group of isometries of a symmetric space of lower dimension than the dimension of $M_{i}$, then $\rho^{*}\left(\varepsilon_{2 n}\right)=0$. Otherwise, it again follows from Lemma 3.1 that $S$ has to be conjugated to a subgroup of the diagonally embedded product $\prod_{i=1}^{n} \mathrm{SL}(2, \mathbb{R})$.

To summarize, we have reduced the proof to the case where the representation $\rho$ factors through

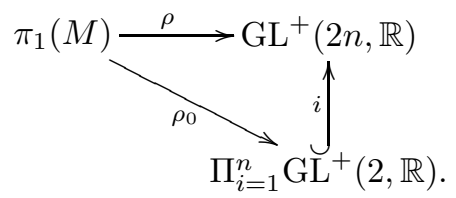


In view of Whitney's product formula for the Euler class, the pullback $i^{*}\left(\varepsilon_{2 n}\right) \in H^{2 n}\left(\prod_{i=1}^{n} \mathrm{GL}(2, \mathbb{R})^{+}\right)$ of the Euler class is the cup product $\varepsilon_{2} \cup \ldots \cup \varepsilon_{2}$ of the Euler class on the factors and hence

$$
\left\|\rho^{*}\left(\varepsilon_{2 n}\right)\right\|_{\infty}=\left\|\rho_{0}^{*}\left(i^{*}\left(\varepsilon_{2 n}\right)\right)\right\|_{\infty}=\left\|\rho_{0}^{*}\left(\varepsilon_{2} \cup \ldots \cup \varepsilon_{2}\right)\right\|_{\infty} \leq\left\|\varepsilon_{2} \cup \ldots \cup \varepsilon_{2}\right\|_{\infty} .
$$

For the simplicial volume of $M$, we show using Hirzebruch's Proportionality Principle [5] that

$$
\|M\|=\frac{|\chi(M)|}{2^{n}\left\|\varepsilon_{2} \cup \ldots \cup \varepsilon_{2}\right\|_{\infty}} .
$$

Finally, combining (2) and (3), and using the duality of the $L^{1}$ and $L^{\infty}$ seminorms (1), we obtain

$$
|\chi(\xi)|=\left\|\rho^{*}\left(\varepsilon_{2 n}\right)\right\|_{\infty}\|M\| \leq \frac{1}{2^{n}}|\chi(M)| .
$$

The details will appear elsewhere.

\section{References}

[1] M. Bucher-Karlsson. The simplicial volume of closed manifolds covered by $\mathbb{H}^{2} \times \mathbb{H}^{2}$, to appear in Journal of Topology.

[2] J.-L. Clerc, B. Ørsted. The Gromov norm of the Kaehler class and the Maslov index. Asian J. Math. 7 (2003), no. 2, 269-295.

[3] A. Domic, D. Toledo. The Gromov norm of the Kaehler class of symmetric domains. Math. Ann. 276 (1987), no. 3, $425-432$.

[4] M. Gromov. Volume and bounded cohomology, Inst. Hautes Études Sci. Publ. Math. No. 56, (1982), 5-99 (1983).

[5] F. Hirzebruch. Automorphe Formen und der Satz von Riemann-Roch, in Symposium Internacional de topologia alebraics, Universidad Nacional Autonoma de Mexico y UNESCO 1958.

[6] N.V. Ivanov, V.G. Turaev, A canonical cocycle for the Euler class of a flat vector bundle. Soviet Math. Dokl. Vol. 26 (1982), No. 1, 78-81.

[7] B. Kostant, D. Sullivan, The Euler characteristic of a compact affine space form is zero, Bull. Amer. Math. Soc. 81, (1974) 937-938

[8] J. Milnor, On the existence of a connection with curvature zero. Comment. Math. Helv. 32 (1958) 215-223.

[9] J. Smillie, Flat manifolds with non-zero Euler characterisitcs, Comment. Math. Helv. 52 (1977) 453-355,

[10] W. Thurston. Geometry and topology of 3-manifolds. Lecture Notes, Princeton (1978).

[11] J. Wood. Bundles with totally disconnected structure group. Comment. Math. Helv. 46 (1971), 257-273. 